\title{
Stability Enhanced AOMDV Protocol for MANETs
}

\author{
Chintan Bhavsar \\ Assistant Professor, \\ G.H Patel College of \\ Engineering \& Technology \\ V. V Nagar, \\ Gujarat, India
}

\author{
Sonal Belani \\ Trainee Assistant Professor, \\ MBICT \\ V. V Nagar, Gujarat, \\ India
}

\begin{abstract}
A Mobile Ad hoc network is set of mobile devices, which communicates without any infrastructure. The topology for such networks is not static because it contains mobile nodes. It also some other limitations like dynamic topology limited and shared bandwidth and limited battery power. Design of a mobile ad hoc network is challenging task, due to such limitations. In such dynamic networks routing is a tough task. Constant movement of nodes increases the possibility of a route failure. Route discovery procedure has to be started to find a new route in case of route failure. As number of route discoveries increases there is an increase in the routing overhead and delay. The existing AOMDV protocol has provision of multiple routes. The route in existing AOMDV is found on minimum hop basis without considering strength of a link. Received signal strength can be used as a metric to find more stable routes. Provision of stable route may increase performance of the network. In this paper performance of stability enhanced AOMDV which considers received signal strength in order to find a route and existing AOMDV protocol are compared.
\end{abstract}

\section{General Terms}

Mobile Ad Hoc Networks

\section{Keywords}

MANET, Routing, Stability, Cross-Layer, Received signal strength, AOMDV.

\section{INTRODUCTION}

A mobile ad hoc network (MANET) is a set of mobile devices connected by wireless links. It is a network which is infrastructure less and dynamic in nature. Each device in MANETs frequently changes its links to other devices as it is free to move any direction. Each node must be equipped to route the traffic. To efficiently route the traffic each device should continuously maintain the routing related information. The mobile nodes of MANETs have limited transmission power which also affects topology of the network [1].

One of the major problems in ad-hoc networking is routing of data packets because, the topology is not predetermined and it does not have centralized control. Hence, routing in ad-hoc networks can be viewed as a challenge due to the frequently changing topology.

There are set of on-demand or reactive routing protocols in MANETs which creates the routes as and when required. Route discovery mechanism is started when there is a requirement to find a route. They only maintain the routes that are currently in use to minimize control overhead and routing load [1].

Existing routing protocols in ad-hoc networks utilize the single route that is built for source and destination node pair.
Due to node mobility, node failures and the dynamic characteristics of the radio channel, links in a route may become temporarily unavailable, making the route invalid [1].

The overhead of finding alternative routes mounts along with additional packet delivery delay. This problem can be solved by use of multiple paths between source and destination node pairs, where one route can be used as the primary route and the rest as backup. Performance can be adversely affected by high route discovery latency and frequent route discovery in dynamic networks. This can be reduced by computing multiple paths in a single route discovery attempt. Multiple paths can be formed for both traffic sources and intermediate nodes with new routes being discovered only when needed, reducing route discovery latency and routing overheads. Multiple paths can also balance network load by forwarding data packets on multiple paths at the same time.

The other way of reducing the routing overhead is to find a route which remains active for longer time. A more stable path could be found that would reduce the number of route failures. A stable route would lead to less number of route discoveries and in turn reduce the routing overhead [15].

We can also find multiple paths such that they are stable. There can be combination of multiple paths with added stability to those paths.

In this paper, we compare two approaches.

(1) Multiple paths in reactive protocol (AOMDV)

(2) AOMDV with Enhanced Stability.

For our purpose we use stability enhanced AOMDV which finds a stable path by considering Received signal strength and AOMDV which is an extension of AODV with provision of multiple paths.

\section{BACKGROUND}

On- demand route discovery is the main principle of reactive routing protocols. On- demand route discovery means creating routes as and when required. They generally find fresh routes, rather than using stored routes. Our discussion is limited to two on-demand ad-hoc routing protocols, AODV and AOMDV, as follows.

\subsection{Ad-hoc On-Demand Distance Vector Routing (AODV)}

AODV is a reactive protocol that discovers routes as and when required. It uses an on-demand route discovery mechanism. It maintains one entry per destination in traditional routing tables. RREQ (route request) packets are broadcasted by sender whenever there is a need to find a route. The RREQ is received by neighboring nodes, which forwards it to their neighbors. RREQ packet travels through 
intermediate node to its intended recipient. Destination node sends RREP (route reply) packet upon receiving RREQ packet. To prevent routing loops and to maintain the freshness of route AODV maintains sequence numbers for each destination [11]. All routing packets carry these sequence numbers. AODV maintains timer based states in each node to remove the unused or older entries in routing table. List of precursors indicates set of neighboring nodes such precursor lists are maintained for each routing table entry. Whenever the next-hop links breaks the nodes in a precursor list are notified by RERR (route error) packet .This Packet gets forwarded by each predecessor node to its Predecessors, effectively erasing all routes using the broken link [11]. The advantages of AODV are that less memory space is required as information of only active routes are maintained, in turn increasing the performance, while the disadvantage is that this protocol is not scalable and in large networks it does not perform well and does not support asymmetric links.

\subsection{Ad-hoc On-demand Multipath Distance Vector Routing (AOMDV)}

Ad-hoc On-demand Multipath Distance Vector Routing (AOMDV) [12] protocol is an extension to the AODV protocol with provision of multiple paths. It finds multiple paths such that they are loop-free and link disjoint paths. There can be multiple next hops for the same destination with same sequence number, which helps in keeping track of a route. AOMDV maintains an advertized hop count for each destination at node. Advertized hop count is nothing but maximum hop count for particular destination. Each duplicate route advertisement received by a node defines an alternate path to the destination. Advertized hop counts ensures Loop freedom for multiple routes. Alternative paths are only considered if they have less hop count than advertized hop count. For same sequence number advertised hop count does not change [12]. The next-hop list and the advertised hop count are reinitialized when a route advertisement is received for a destination with a greater sequence number.

AOMDV finds multiple paths which are node-disjoint or link disjoint routes. Each RREQs arriving via a different neighbor of the source defines a node-disjoint path. To find nodedisjoint routes, each node does not immediately reject duplicate RREQs. This is because nodes cannot broadcast duplicate RREQs, so any two RREQs arriving at an intermediate node via a different neighbor of the source could not have traversed the same node. Destination only replies to RREQs arriving via unique neighbors in an order to get multiple link-disjoint routes. After the first hop, the RREPs follow the reverse paths, which are node disjoint and thus link-disjoint. Each RREP may intersect at an intermediate node, but each takes a different reverse path to the source to ensure link disjointness [12]. The advantage of using AOMDV is that it allows intermediate nodes to reply to RREQs, while still selecting disjoint paths. As AOMDV finds multiple paths and destination replies to multiple RREQs it has more routing overhead during route discovery.

\section{RELATED WORK}

\subsection{Approaches to find Stable Path}

There are number of approaches to find a stable or reliable route for ad hoc networks. These approaches have different parameters like node residual energy, link expiration time, Stability of nodes, probabilistic link lifetime estimation and success rate of data transmission for node, link usage, and received signal strength [2].

\subsection{Using Received Signal Strength for Stable Route}

Existing on demand routing protocols for ad hoc network does not take in its consideration the quality of link while deciding the route. If decision for selecting a route is based on received signal strength then the protocols can achieve the route which is more stable. Simulation in [6] shows good resemblance with actual implementation. Higher packet delivery ratio and lower routing load can be achieved if received signal strength is used as a parameter for finding the route [4] [15].

\subsection{Cross- Layer Design}

In a cross layer design the parameter measured at physical layer can be made available to layers above it. The value measured at physical layer is passed to MAC layer and network layer. The received signal strength information sent by the physical layer may be stored either in routing table or neighbor table and can be used to make routing decisions. Such a cross layer design has been utilized to improve the performance of mobile ad hoc network [14].

Received signal strength of RREQ packet can be measured at physical level and based on that routing decisions can be made at routing layer [4]. If RREQ packet has a poor received signal strength then receiving node should not forward the RREQ to its neighbors, so weak link does not participate in the route. The received signal strength can be compared with a predetermined threshold to determine whether it is proper or not. The threshold can be a fixed threshold or an adaptive threshold whose value changes with changing speed of the nodes. The adaptive threshold also adapts to moving direction of the nodes.

The path loss of the link can be found by measuring the received signal strength by cross-layer approach [3]. The path loss measured for each link of an entire route can be added to find cumulative path loss of entire route. The average path loss for route can be found by dividing total path loss with hop count. Only the route which is having less path loss can be selected, so resulting route can be more stable.

The AODV-RSS in [5] determines paths that are long lived means, a route that can sustain for a longer time. The longlived path routing algorithm uses the Received Signal Strength RSS and Received Signal Strength changing rate $\Delta$ RSS to predict the link available time (LAT) between two mobile nodes. AODV-RSS can improve the route quality in route connection time, and route reestablishment frequency.

The Route Stability based QOS Routing (RSQR) protocol proposed in [7] which is an extension of QOS routing with throughput and delay constraints. In order to guarantee the suitable data path for adequate longer duration in MANET, an easy model has been proposed for measuring the link stability and route stability depending on received signal strength. Some additional fields in route request/ reply packets is taken into consideration so that the route stability information can be used to choose a route with increased Stability when compared to all possible routes among existing source destination pair.

\section{STABILITY ENHANCED AOMDV PROTOCOL}

AOMDV is an on-demand routing protocol which broadcast RREQ (route request) packet to all neighbors in order to determine the route. Intermediate nodes checks destination address and accordingly they forward RREQ to their 
neighbors. A RREQ packet traversing from different path arrives at the destination. RREP (route reply) packet is send from receiver upon receiving RREQ [12]. AOMDV finds multiple paths for a single source destination pair. Those routes may have weak links which may lead to frequent route failures [6]. Frequent route failures will increase the number of route discoveries and in turn increase the routing overhead of the network.

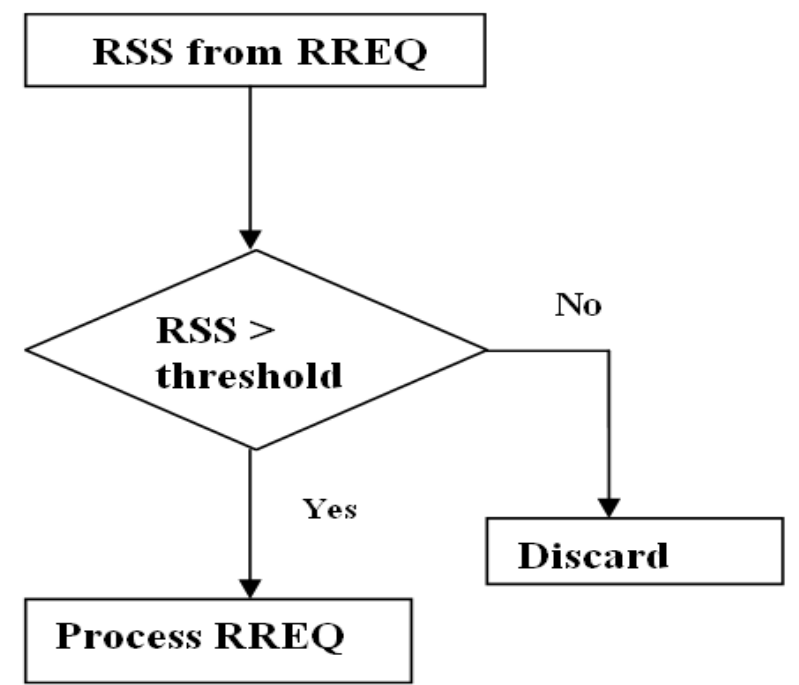

Fig. 1: Processing RREQ in Stability enhanced AOMDV

Minimization of routing overhead of the network is primary focus of the stability enhanced AOMDV protocol. The received signal strength of the link is determined by measuring received signal strength of RREQ packet. Received signal strength information measured at physical layer is used at routing layer through cross layer design. A RREQ packet is only forwarded if the link has sufficient received signal strength. So, the links with lower received signal strength i.e. weak links may not participate in formation of route.

A predetermined threshold is used to decide whether the link has the sufficient received signal strength or not. The threshold to be used can be a fixed value which can be determined based on performance.

As shown in Figure 1 stability enhanced AOMDV protocol compares received signal strength of RREQ with predetermined threshold. The threshold value is nothing but the desired value of the received signal strength for link. If the received signal strength from the RREQ is greater than the threshold then RREQ packet will be processed else it will be discarded. In this way it takes care that no weak links can participate in formation of route. The route found by this method is stable and remains active for longer time. With added stability to the route number of route failures can be reduced which can help in reducing routing load of the network. The method which is applied here finds the route which can contains increased number hops compared to route in existing AOMDV. The increase in number of hops can also increase end to end delay of the network. An additional delay can also affect throughput of the network.

\section{SIMULATION ENVIRONMENT AND RESULT ANALYSIS}

Network simulator 2 is a simulation tool which has been used to simulate both stability enhanced AOMDV and existing AOMDV protocol. NS-2.34 version is used which has support for simulating a multi-hop wireless ad-hoc environment.49 nodes are deployed in an area of $1000 \times 1000 \mathrm{~m} 2$. Each point in result is an average of 10 seeds. The other simulation parameters are summarized in table I.

We tested following two cases

1) Stability Enhanced AOMDV

2) AOMDV.

The fixed threshold value of 200 (received signal strength which is measured after traveling 200 meters) is used for "Stability Enhanced AOMDV" and it is tested for different speed in the range of $5 \mathrm{~m} / \mathrm{s}-25 \mathrm{~m} / \mathrm{s}$.

Table 1. Simulation Parameters

\begin{tabular}{cc}
\hline Parameters & Values \\
\hline Transmission range & 250 meter \\
Mobility model & Random Way point \\
Propagation model & Two Ray Ground \\
MAC Layer & 802.11 \\
Simulation time & $200 \mathrm{sec}$ \\
Transmission protocol & TCP \\
Routing protocol & Stability enhanced \\
& AOMDV, AOMDV \\
Packet size & 512 Bytes \\
\hline
\end{tabular}

\subsection{Packet Delivery Ratio}

The packet delivery ratio is no of packets received per number of packet sent. Table 2 lists simulation results of packet delivery ratio obtained for stability enhanced AOMDV and AOMDV against various speed of nodes ranging from 5 meter/second to 25 meter/second. Each result in Table 2 is an average of 10 seeds. Fig. 2 shows the packet delivery ratio for stability enhanced AOMDV and AOMDV. The stability enhanced AOMDV seem to achieve better performance than AOMDV in terms of packet delivery ratio.

Table 2. Simulation results for Packet delivery ratio

\begin{tabular}{lcc}
\hline Speed & AOMDV & Stability Enhanced AOMDV \\
\hline $\mathbf{5 ~} \mathbf{~} / \mathbf{s}$ & 0.9865 & 0.9870 \\
$\mathbf{1 0} \mathbf{~} / \mathbf{s}$ & 0.9750 & 0.9765 \\
$\mathbf{1 5} \mathbf{~ m} / \mathbf{s}$ & 0.9505 & 0.9607 \\
$\mathbf{2 0} \mathbf{~} / \mathbf{s}$ & 0.9615 & 0.9668 \\
$\mathbf{2 5} \mathbf{~ m} / \mathbf{s}$ & 0.9721 & 0.9769 \\
\hline
\end{tabular}

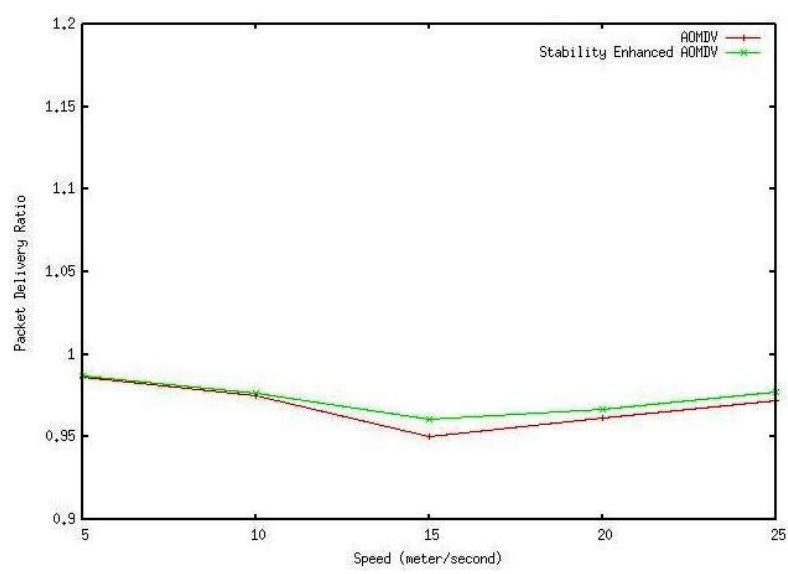

Fig. 2: Shows packet delivery ratio against speed of nodes 


\subsection{Normalized Routing Load}

The normalized routing load is number of routing packets sent per number of data packet received. Table 3 lists simulation results of Normalized Routing Load obtained for stability enhanced AOMDV and AOMDV against various speed of nodes ranging from 5 meter/second to 25 meter/second. Each result in Table 3 is an average of 10 seeds. From Fig 3 it can be observed that AOMDV has more routing overhead compared to stability enhanced AOMDV. Stability enhanced AOMDV do not process the RREQ if it is having lower received signal strength, which will reduce the routing load in stability enhanced AOMDV.

Table 3. Simulation results for Normalized Routing Load

\begin{tabular}{lcc}
\hline Speed & AOMDV & Stability Enhanced AOMDV \\
\hline $\mathbf{5} \mathbf{~} / \mathbf{s}$ & 1.267 & 1.213 \\
$\mathbf{1 0} \mathbf{~} / \mathbf{s}$ & 2.224 & 2.134 \\
$\mathbf{1 5} \mathbf{~} / \mathbf{s}$ & 6.983 & 3.937 \\
$\mathbf{2 0} \mathbf{~} / \mathbf{s}$ & 4.631 & 4.095 \\
$\mathbf{2 5} \mathbf{~} / \mathbf{s}$ & 3.541 & 1.878 \\
\hline
\end{tabular}

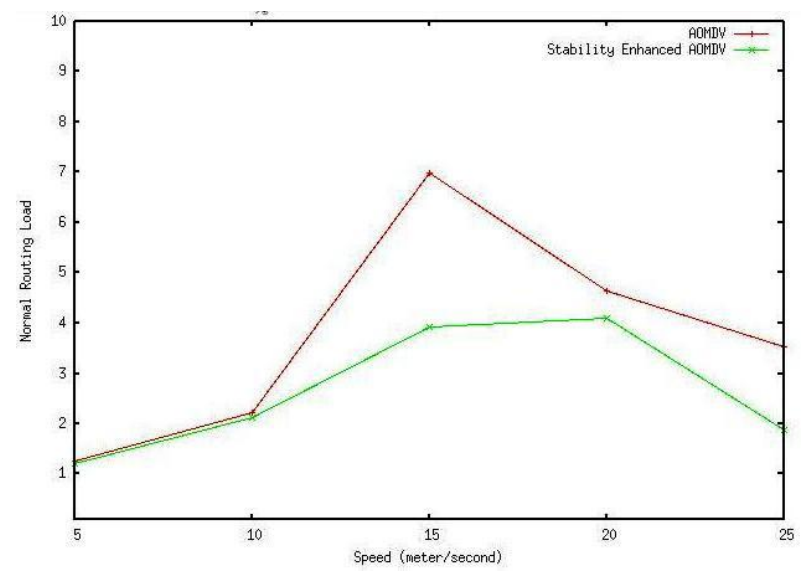

Fig. 3: Shows normalized routing load against speed of nodes

\subsection{Average End To End Delay}

The average end to end delay is the average of delay occurred in transmitting each data packet. Table 4 lists simulation results of Average End to End Delay obtained for stability enhanced AOMDV and AOMDV against various speed of nodes ranging from 5 meter/second to 25 meter/second. Each result in Table 4 is an average of 10 seeds. Fig 4 shows that AOMDV has lower average end to end delay compared to stability enhanced AOMDV. The approach adapted by stability enhanced AOMDV increases number of hops in the route which can cause an additional delay.

Table 4. Simulation results for Average End ToEnd Delay

\begin{tabular}{lcc}
\hline Speed & AOMDV & Stability Enhanced AOMDV \\
\hline $\mathbf{5} \mathbf{~} / \mathbf{s}$ & 243.334 & 263.873 \\
$\mathbf{1 0} \mathbf{~} / \mathbf{s}$ & 235.55 & 260.117 \\
$\mathbf{1 5} \mathbf{~} / \mathbf{s}$ & 154.898 & 197.525 \\
$\mathbf{2 0} \mathbf{~} / \mathbf{s}$ & 225.922 & 239.652 \\
$\mathbf{2 5} \mathbf{~ m} / \mathbf{s}$ & 116.106 & 199.66 \\
\hline
\end{tabular}

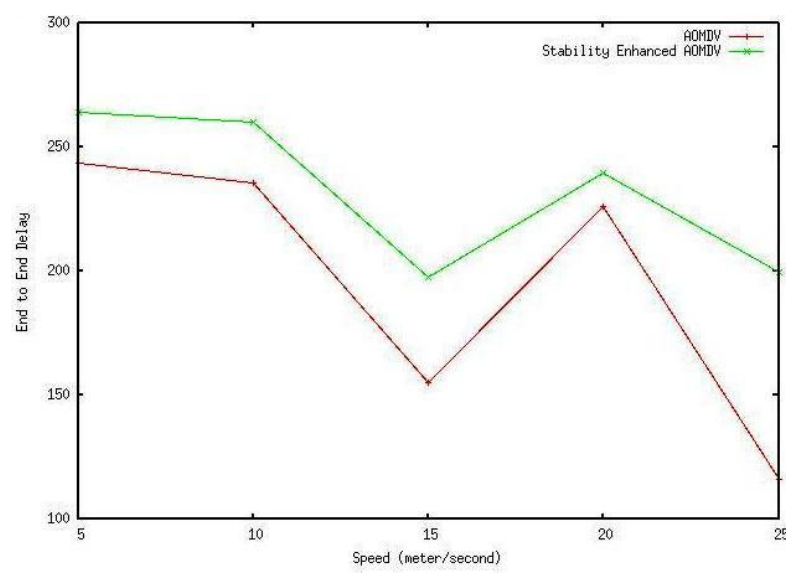

Fig. 4: Shows end to end delay against speed of nodes

\subsection{Throughput}

The throughput is total data transmitted per second. Table 5 lists simulation results of Throughput obtained for stability enhanced AOMDV and AOMDV against various speed of nodes ranging from 5 meter/second to 25 meter/second. Each result in Table 5 is an average of 10 seeds. It can be observed form Fig 5 that stability enhanced AOMDV achieve higher throughput than AOMDV. Stability enhanced AOMDV has higher end to end delay but it has lower routing load compared to AOMDV which increases the throughput.

Table 5. Simulation results for Throughput

\begin{tabular}{lcc}
\hline Speed & AOMDV & Stability Enhanced AOMDV \\
\hline $\mathbf{5} \mathbf{~ m} / \mathbf{s}$ & 210.396 & 227.169 \\
$\mathbf{1 0} \mathbf{~} / \mathbf{s}$ & 148.395 & 149.861 \\
$\mathbf{1 5} \mathbf{~} / \mathbf{s}$ & 132.812 & 137.538 \\
$\mathbf{2 0} \mathbf{~} / \mathbf{s}$ & 75.64 & 78.227 \\
$\mathbf{2 5} \mathbf{~} / \mathbf{s}$ & 102.696 & 137.91 \\
\hline
\end{tabular}

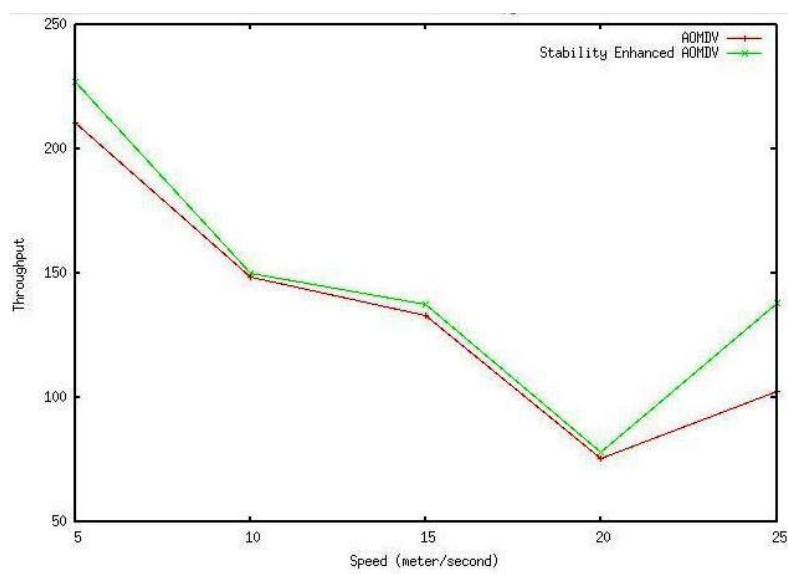

Fig. 5: Shows throughput against speed of nodes

\section{CONCLUSION}

In this paper performance of stability enhanced AOMDV and AOMDV protocol are compared. Results suggest that stability enhanced AOMDV has lower routing load compared to AOMDV protocol. Provision of stable path in stability enhanced AOMDV reduces number of route failures, which will also reduce number of route discovery as well as routing load.

In stability enhanced AOMDV unnecessary RREQ packets are dropped which also reduces the routing load of the 
network. Due to reduced routing load stability enhanced AOMDV achieves better packet delivery ratio and throughput than AOMDV.

It can also be observed that stability enhanced AOMDV has higher End to End delay compared to AOMDV protocol.

Stability enhanced AOMDV increases number of hop in route compared to AOMDV. Increased number of hops in route is also increases end to end delay.

Reduced routing load, higher packet delivery ratio and higher throughput can be achieved by provision of stable route in AOMDV.

\section{REFERENCES}

[1] H.D.Trung, W.Benjapolakul, P.M.Duc, May 2007 "Performance evaluation and comparison of different ad hoc routing protocols", Department of Electrical Engineering, Chulalongkorn University, Bangkok, Thailand.

[2] Hadi Sargolzaey, Ayyoub Akbari Moghanjoughi and Sabira Khatun, January 2009," A Review and Comparison of Reliable Unicast Routing Protocols For Mobile Ad Hoc Networks", International Journal of Computer Science and Network Security (IJCSNS), VOL. 9 No.1.

[3] Boumedjout Amel, Mekkakia Maaza Zoulikha, "Routing Technique with Cross-layer Approach in Ad Hoc Network”, IEEE, 2009

[4] B.Ramchandran and S. shanmugavel, JUL-AUG 2008,"Received Signal Strength-based Cross-layer design for Mobile Ad Hoc Networks", IETE Technical Review, Volume 25, Issue 4.

[5] Ruay-Shiung Chang and Shing-Jiuan Leu, January 2006,"Long-lived Path Routing With Received Signal Strength for Ad Hoc Networks", IEEE International Symposium on Wireless Pervasive Computing.

[6] Kwan-Wu Chin, John Judge, Aidan Williams and Roger Kermode, November 2002,'Implementation Experience with MANET Routing Protocols", ACM SIGCOMM
Computer Communications Review, Volume 32, Number 5.

[7] Nityananda Sharma and Sukumar Nandi, "Route Stability Based QoS Routing in Mobile Adhoc Networks", Wireless Personal Communication, Vol-54, pp-203-224, 2010.

[8] Fang Xie, Lei Du, Yong Bai, Lan Chen, "Energy Aware Reliable Routing Protocol for Mobile Ad-HocNetworks", IEEE Communication Society, WCNC 2007proceedings.

[9] Zhao Cheng, Wendi B. Heinzelman, "Discovering long lifetime routes in mobile ad hoc networks", Elsevier Ad Hoc Networks 6, 2008, p661-674.

[10] Xie Xiaochuan, Wei Gang, Wu Keping, Wang Gang, Jia Shilou, "Link reliability based hybrid routing for tactical mobile ad hoc network", Elsevier Journal of Systems Engineering and Electronics Vol. 19, No. 2, 2008, pp.259-267.

[11] Charles E. Perkins, Elizabeth M. Belding-Royer, Samir R. Das, February 2003, "Ad hoc On-Demand Distance Vector (AODV) Routing draft-ietf-manet-aodv-13.txt,", Mobile Ad Hoc Networking Working Group, INTERNET DRAFT.

[12] M.K.Marina and S.R.Das, 2001, "On-Demand Multipath distance vector routing in ad hoc Networks" in: Proceedings of 9th IEEE International Conference on Network Protocols (ICNP).

[13] The ns Manual, Formerly ns Notes and Documentation.

[14] Chintan Bhavsar,"A Survey On Cross-Layer Reliable Routing Protocols in MANETs", International Journal of Innovative Research in Science, Engineering and Technology, Volume 03, Issue 4, April 2014.

[15] Chintan Bhavsar, Mayur M. Vegad, Sunil A. Bakhru, "Performance Comparison of Stability Enhanced AODV and AOMDV protocols for MANETs", IJETAEInternational Journal of Emerging Technology and Advanced Engineering, Volume 2, Issue 5, May 2012. 